Bull. Austral. Math. Soc.

39A11, 92D25

VOL. 58 (1998) [169-171]

\title{
Stability of nonlinear discrete systems with applications to population dynamics
}

\author{
Pingzhou LiU
}

Applications of differential equations to population dynamics have been widely studied. At present, there has been a steady increase in the interest on difference equations as they are playing a fundamental role in our understanding of nonlinear phenomena. Simplicity for computation and complexity of dynamical behaviour are two distinctive features of difference systems. The equations with discrete time usually arise when values under consideration are measured after certain intervals of time. One can either design a discrete time model from a original real problem or one can discretize known differential equations. The second approach has the advantage that one can keep track of the known properties of the continuous time system. Of course, taking differential equations as the main "source" of difference equations, one should check whether the equations still have the desired properties as their continuous counterparts. In the literature, the study and analysis of differential equations (continuous time) and difference equations (discrete time) have not been treated in a unified format. The main aim of this thesis is to initiate a systematic study of the relation between differential dynamic systems and their discrete analogues, especially for systems which have been widely used in modelling the dynamics of single and several interacting species of populations. As the applications of differential equations to population dynamics are quite fruitful (see, for example [1, 2, 7]), through the analogous difference equations we may find some new applications of difference equations. On the other hand, we may use the analogous difference equations to get a "best" approximation to a given differential dynamic system. We believe the results obtained are mathematically interesting and lead to a better understanding of population dynamics especially of those species with nonoverlapping generations. Similar ideas can be extended to economic systems and control systems.

As a linear analysis is the first step to deal with nonlinear systems, we discuss in Chapter 1, linear systems of Volterra difference equations and include most techniques

Received 9th March, 1998

Thesis submitted to The Flinders University of South Australia August 1997. Degree approved December 1997. Supervisor: Associate Professor K. Gopalsamy.

Copyright Clearance Centre, Inc. Serial-fee code: 0004-9729/98 \$A2.00+0.00. 
for linear analysis. In Chapter 2 we consider a class of difference equations with infinitely many delays which gives a "best" approximation of a logistic integrodifferential equation. The third Chapter deals with a differential equation having piecewise constant arguments. As an application, a thorough analysis of logistic dynamics under harvesting is developed; both local and global stability of a positive equilibrium under harvesting are studied. We also show the complexity of its behaviour for certain regions in the parameter space.

A model of social population is studied in Chapter 4. The results include numerical simulations and potential chaotic behaviour of the model.

In Chapter 5, the discrete dynamics of Lotka-Volterra systems of two species are investigated. This investigation continues in Chapter 6 for the higher dimensional competition systems in periodic environments. The existence of a stable periodic solution is established.

Some results of this thesis have been written as publications $[3,4,5,6,8,9,10,11]$.

\section{REFERENCES}

[1] H.I. Freedman, Deterministic mathematical models in population ecology (Marcel Dekker Inc., New York, 1980).

[2] K. Gopalsamy, Stability and oscillations in delay differential equations of population dynamics (Kluwmer Academic, Dordrecht, The Netherlands, 1992).

[3] K. Gopalsamy and P. Liu, 'On a discrete model of mutualism', Proceeding of 3rd ICDEA, Academia Sinica, Taipei (to appear).

[4] K. Gopalsamy and P. Liu, 'Dynamics of Social populations, Nonlinear Analysis, Theory, Methods and Applications', Proceeding of the Second World Congress of Nonlinear Analysis, Athens, Greece 30 (1996), 2595-2604.

[5] K. Gopalsamy and P. Liu, 'Persistence and global stability in a population model', $J$. Math. Anal. Appl. (in press).

[6] K. Gopalsamy and P. Liu, 'Dynamics of a logistic map with eventually fading memory', Dynamic Systems Appl. 6 (1997), 1-10.

[7] Y. Kuang, Delay differential equations with applications in population dynamics (Academic press, New York, 1993).

[8] P. Liu and K. Gopalsamy, 'Dynamics of a hyperbolic logistic map with fading memory', Dynam. Contin. Discrete. Impuls. Systems 1 (1995), 53-67.

[9] P. Liu and K. Gopalsamy, 'On a population model which satisfies Allee's principle', Proceedings of The International Conference On Mathematical Biology, Hangzhou, China (1997).

[10] P. Liu and K. Gopalsamy, 'On a model of competition in periodic environments', Appl. Math. Comput. 82 (1997), 207-238.

[11] P. Liu and K. Gopalsamy, 'Global stability and chaos in a population model with piecewise constant arguments', Appl. Math. Comput. (to appear). 
Department of Maths and Statistics The Flinders University of South Australia GPO Box 2100

Adelaide SA 5001

Australia 